\title{
Identification of microorganisms in irreversible pulpitis and primary endodontic infections with respect to clinical and radiographic findings
}

\author{
Nazanin $\operatorname{Zargar}^{1} \cdot$ Hengameh Ashraf $^{1}$ - S. M. Amin Marashi ${ }^{2} \cdot$ Mohammad Sabeti $^{3} \cdot$ Alireza Aziz $^{4}$ \\ Received: 23 February 2019 / Accepted: 13 September 2019 / Published online: 6 May 2020 \\ (C) Springer-Verlag GmbH Germany, part of Springer Nature 2019
}

\begin{abstract}
Objectives The aim of this study was to evaluate the composition of microbiota of irreversible pulpitis and primary endodontic infections with respect to clinical and radiographic findings by performing cultures and $16 \mathrm{~s}$ rDNA sequencing in Iranian patients. Material and methods In this prospective cross-sectional study, samples were collected from 41 root canals for 4 main groups of patients. Bacterial identification was performed by the polymerase chain reaction (PCR) and 16s rDNA sequencing of aerobic and anaerobic cultivable colonies taken from patients' culture plates. Additionally, the presence of 13 bacterial species and 3 nonbacterial species was also explored using PCR and species-specific primers.

Results Sixteen microbial species, 1 fungus (Candida albicans), and 1 virus (Herpes simplex virus) were discovered and isolated. Species with the highest prevalence were Dialister invisus (68.3\%), Porphyromonas gingivalis (58.8\%), Streptococcus salivarius (58.5\%), and Treponema denticola (56.1\%). Lysinibacillus fusiformis (19.1\%) was detected in the root canals for the first time. Candida albicans was seen in 11 cases (26.8\%). Herpes simplex virus (HSV) was seen in 4 patients $(9.8 \%)$.

Conclusions Our results suggest that Gram-negative anaerobic oral bacteria are the majority of the microbes in primary endodontic infections. Various combinations of bacterial species were related to different clinical and radiographic conditions. Lysinibacillus fusiformis was detected for the first time in primary endodontic infections.

Clinical relevance The results of this investigation might help clinicians choose to identify suspected endodontic pathogens in the etiology of each form of pulpal and periradicular diseases to determine the best therapeutic measures.
\end{abstract}

Keywords Bacterial identification $\cdot$ Polymerase chain reaction (PCR) $\cdot$ Primary endodontic infection $\cdot 16 \mathrm{~s}$ rDNA

\section{Introduction}

Bacterial agents are generally accepted to be the main causes of endodontic infections and failure. Few species have been associated with treatment failures [1-3]. Microorganisms penetrate the dentin and ultimately the pulp via deep caries or

Alireza Aziz

alireza_az91@yahoo.coms

Nazanin Zargar

nzargar48@yahoo.com

Hengameh Ashraf

he_ashraaf@yahoo.com

S. M. Amin Marashi

parsmicrob@gmail.com

Mohammad Sabeti

Mike.sabeti@ucsf.edu cracks and may enter the periodontal tissue or the pulp directly as a result of an iatrogenic restorative procedure or trauma; such conditions ultimately lead to necrosis and infection of the pulp [4].

In previous studies, bacteria involved in endodontic infections were mostly identified by microbial culture methods [5].

1 Department of Endodontics, School of Dentistry, Shahid Beheshti University of Medical Sciences, Daneshjoo Blv., Daneshgah Square, Chamran Highway, Tehran 1983963113, Iran

2 Medical Microbiology Research Center, Qazvin University of Medical Sciences, Qazvin, Iran

3 UCSF School of Dentistry, 707 Parnassus Ave. Room-D 3226, San Francisco, CA 94143-0758, USA

4 School of Dentistry, Shahid Beheshti University of Medical Sciences, Daneshjoo Blv., Evin Avenue, Chamran Highway,

Tehran 1983963113, Iran 